\title{
Zukunft der ärztlichen Psychotherapie in der Psychiatrie
}

\author{
Future of Medical Psychotherapy in Psychiatry
}

Autoren

Institute
Sabine Herpertz ${ }^{1}$, Peter Falkai ${ }^{2}$

Klinik für Allgemeine Psychiatrie, Zentrum für Psychosoziale Medizin, Universitätsklinikum Heidelberg

Abteilung für Psychiatrie und Psychotherapie, Universitätsmedizin Göttingen

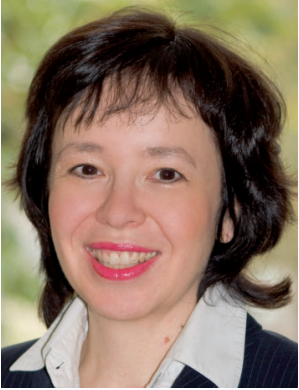

Prof. Dr. Sabine Herpertz

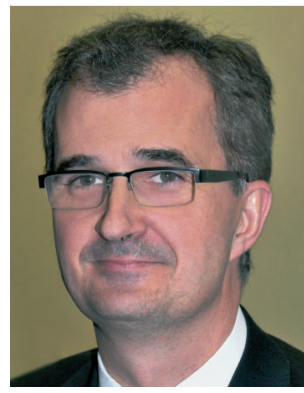

Prof. Dr. Peter Falkai

\section{Bibliografie}

Dol http://dx.doi.org/ 10.1055/s-0032-1304943 Psychiat Prax 2012; 39:

151-152

(c) Georg Thieme Verlag KG

Stuttgart · New York

ISSN 0303-4259

Korrespondenzadresse

Prof. Dr. Peter Falkai

Abteilung für Psychiatrie und Psychotherapie,

Universitätsmedizin Göttingen

von-Siebold-Straße 5

37075 Göttingen

Peter.Falkai@medizin.uni-

goettingen.de
Psychiatrische Erkrankungen sind beim überwiegenden Teil der Betroffenen gekennzeichnet durch Phasen mit klar erkennbarer und erfahrbarer Symptomatik sowie Phasen der weitestgehenden Rückbildung des Beschwerdebilds. Dementsprechend unterscheidet man zwischen einer ca. 4-wöchigen Akutphase, einer etwa 3-monatigen Postakutphase, gefolgt von einer Remissionsund Unterhaltungsphase.

Psychische Erkrankungen sind auch komplexe Störungen, bei denen anlagebedingte Faktoren und Umweltfaktoren zum Krankheitsbild beisteuern. Diese Komplexität fordert in der Regel die Anwendung verschiedener Therapien, sei es hintereinander oder parallel. Ein paradigmatisches Beispiel hierfür sind die affektiven Störungen und hier die unipolare Depression, wo bereits mit der mittelgradigen Episode Psycho- und Pharmakotherapie kombiniert angeboten werden müssen [1].

Für die Psychotherapie der affektiven Erkrankungen stehen eine Reihe wissenschaftlich evaluierter störungsspezifischer Methoden zur Verfügung, so z.B. für die episodische Depression die Kognitive Verhaltenstherapie (KVT) oder die Interpersonelle Psychotherapie (IPT) und für die chronische Depression das Cognitive Behavioral Analysis System of Psychotherapy (CBASP). Auch liegen für die Psychodynamische Kurzzeittherapie bei akuten Depressionen Wirksamkeitsstudien vor. In der ambulanten Behandlung aber wird Psychotherapie im Rahmen der sog. Richtlinienverfahren durchgeführt, die somit per definitionem kognitiv-verhaltenstherapeutisch, tiefenpsychologisch oder analytisch begründet sein müssen und damit nicht schulenintegrativ konzipierte, aber empirisch am besten validierte störungsorientierte Methoden wie IPT und CBASP abbilden. Auch wenn dieses etablierte Vorgehen Vorteile haben mag, führt es dazu, dass es aufgrund der Wartezeit und des hiermit verbundenen Leidensdrucks in der Regel nur teilremittier- ten Patienten mit einer affektiven Erkrankung zugute kommt ( $\bullet$ Abb. 1). Zudem wurden Richtlinienverfahren nicht als Teil eines multimodalen Vorgehens konzeptionalisiert und sehen auch kein flexibles, verfahrenübergreifendes Vorgehen in Abhängigkeit vom Krankheitsbild und weiteren Patientenmerkmalen vor.

Trotz weitgehend stabiler Prävalenz wächst die Nachfrage nach Behandlung psychischer Erkrankungen stetig, vermutlich aufgrund verminderter Stigmatisierung, aber auch durch früheres Erkennen und Behandeln psychischer Erkrankungen. Wie kann die Psychiatrie dem offensichtlich steigenden Bedarf nach Psychotherapie einerseits, der Notwendigkeit, Psychotherapie pragmatisch in den verschiedenen psychischen Erkrankungsphasen anzubieten anderseits, begegnen? Bereits heute schon können Fachärzte für Psychiatrie und Psychotherapie bzw. Nervenheilkunde bei über 400 Behandlungsfällen im Quartal und damit einer 8-fach höheren Fallzahl im Vergleich zu ausschließlich psychotherapeutisch tätigen Ärzten und Psychologen dem psychotherapeutischen Bedarf ihrer Patienten nicht gerecht werden.

Unserer Meinung nach gibt es mittlerweile genügend evidenzbasierte störungsspezifische Psychotherapien für den Bereich der affektiven Erkrankungen, Suchterkrankungen, psychotischen Störungen, Persönlichkeitsstörungen und vielen anderen, die den Betroffenen in jeder Phase einer psychischen Erkrankung eine adäquate psychotherapeutische Behandlung bieten könnten. Vor allem eine modulare Psychotherapie könnte eine Antwort darstellen, wie psychotherapeutische Interventionen flexibel und passgenau auf die Bedürfnisse genau der Patienten angepasst werden, die sich typischerweise dem Psychiater vorstellen. Modulare Psychotherapie gibt einerseits eine pragmatische Gliederung der derzeit etablierten therapeutischen Strategien und Interventionen wieder und stellt andererseits eine Handreichung in Form von Heurismen dar, die vorgibt, welche 


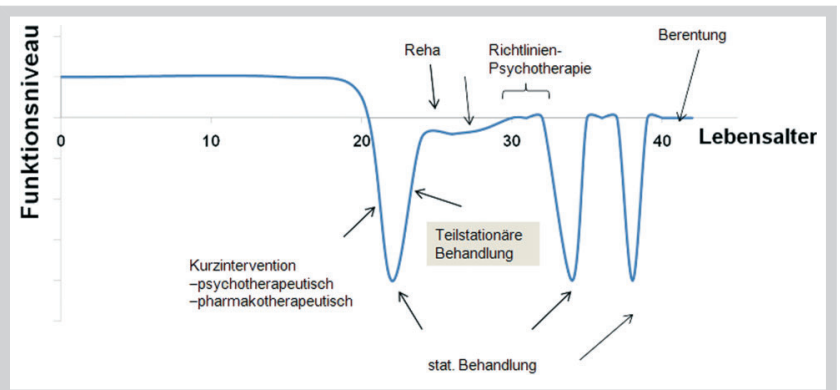

Abb.1 Verlauf psychischer Erkrankungen [2].

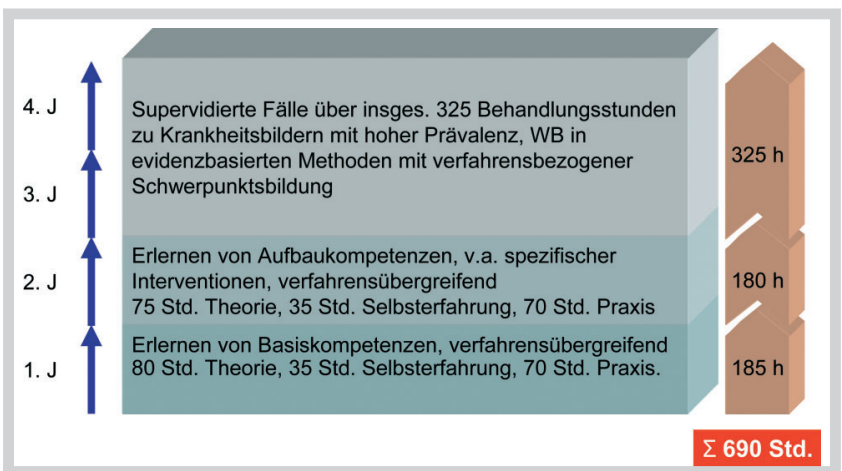

Abb.2 Modulare psychiatrische Weiterbildung im Ausblick [2].

dieser Strategien im Einzelfall zur Anwendung kommen sollten. Für eine Verbesserung der Versorgungssituation auch in Zeiten steigenden Bedarfs könnte eine stärkere Verankerung dieser modularen Psychotherapie in der Weiterbildung zum Facharzt für Psychiatrie und Psychotherapie interessant sein. Assistenzärzte könnten so von Anfang an die wesentlichen Basisfertigkeiten, Strategien, Interventionen und Heurismen zunächst theoretisch und dann v.a. in der Anwendung unter Nutzung videobasierter Supervisionstechniken und Rollenspielen erlernen. Trotz klarer Darlegung der zu vermittelnden psychotherapeutischen Inhalte in der aktuellen Weiterbildungsordnung fehlt eine Verzahnung der theoretischen Weiterbildung mit der klinischen Anwendung im derzeitigen Alltag. Zumindest scheint zwar weitläufig eine theoretische Vermittlung der Grundlagen der Psychotherapie durchaus zu erfolgen, an einer ausreichenden Anwendung des Erlernten in der klinischen Praxis scheint es jedoch zu mangeln. Aus diesem Grund hat die DGPPN sich entschlossen, eine Arbeitsgruppe Psychotherapie unter Leitung von Sabine Herpertz einzurichten. Zu dieser gehören M. Linden (Berlin), H. Freyberger (Greifswald), M. Bohus (Mannheim/Heidelberg), M. Urban (Berlin) und P. Falkai (Göttingen). Ziel dieser Arbeitsgruppe war der Entwurf eines neuen Curriculums für eine modulare psychiatrische Psychotherapie, die eine stärkere Verschränkung zwischen theoretischen Vermittlungen psychotherapeutischer Inhalte und der Anwendung in der klinischen Praxis erlaubt ( $\bullet$ Abb.2).

Demzufolge wäre es wünschenswert, im 1. Jahr verfahrensübergreifende Basiskompetenzen zu vermitteln und im 2. Jahr spezifische Interventionen zu erarbeiten und mit den Weiterbildungskandidaten zu üben. Im 3. und 4. Weiterbildungsjahr sollten bei den Krankheitsbildern mit hoher Prävalenz evidenzbasierte Psychotherapiemethoden mit verfahrensbezogener Schwerpunkt- bildung vermittelt werden. Zum Erlernen der störungsspezifischen Psychotherapiemethoden für wesentliche Krankheitsbilder liegt nun ein detaillierter Vorschlag der Arbeitsgruppe vor. Hiernach sollten Weiterbildungskandidaten die Verfahren so eingehend wie möglich erlernen, um im Bereich von Richtlinienverfahren tätig werden zu können, damit sich psychotherapeutische Leistungen in diesem Rahmen auch abrechnen lassen.

Eine weitere wesentliche Forderung der Deutschen Gesellschaft für Psychiatrie, Psychotherapie und Nervenheilkunde (DGPPN) ist in diesem Zusammenhang eine adäquate Supervision der praktischen psychotherapeutischen Weiterbildung. Nach Wunsch der Fachgesellschaft begleitet ein Fallführungssupervisor jede 4. Stunde mittels Videoaufzeichnung oder videogestützter Direktsupervision der Aktivitäten im Einzel- oder Gruppensetting der Weiterbildungskandidaten. Hierfür gilt es aus Sicht der DGPPN, die Oberarztkompetenz mit einer Supervisionsausbildung zu verknüpfen, um eine qualitative hochwertige Supervision der psychotherapeutischen Weiterbildung zu ermöglichen.

Ziel sollte ein psychotherapeutisch breitflächig ausgebildeter Facharzt für Psychiatrie und Psychotherapie für die Behandlung des Gesamtkanons der psychischen Erkrankungen sein. Diese Prämisse gilt für die Akutphase, in welcher primär psychoedukative und stützende Elemente überwiegen, gefolgt von der Postakutphase, wo eine störungsspezifische Psychotherapiemethode systematisch Anwendung findet, bis hin zu einer psychotherapeutischen Unterstützung in der Erhaltungsphase, welche sich primär auf die Verhinderung eines Rezidivs ausrichtet. Dieser Ansatz sollte gewährleisten, dass möglichst viele Menschen mit psychischen Erkrankungen, insbesondere solche mit komorbiden somatischen und psychischen Erkrankungen, eine pragmatische, schnelle und evidenzbasierte Therapie erhalten. Dies erfordert natürlich, dass neben den Richtlinienverfahren auch mindestens 20-minütige, wenn nötig auch längere psychotherapeutische Interventionen in unterschiedlichen Phasen einer psychischen Erkrankung adäquat abgerechnet werden können. So ließe sich eine wirkliche psychotherapeutische Intervention von einer kurzen verbalen Interaktion trennen, eröffnete aber auch die Möglichkeit, z.B. bei beginnendem Rezidiv einer affektiven Erkrankung eine Serie von psychotherapeutischen Interventionen einzuleiten, um den Ausbruch der Erkrankung zu verhindern. In der täglichen Praxis findet dies heute bereits statt, lässt sich aber nicht adäquat abrechnen. Aus diesem Grunde suchen viele gut ausgebildete Fachärzte für Psychiatrie und Psychotherapie den Weg in die reine Psychotherapiepraxis, worunter die breit ausgerichtete Basisversorgung im Bereich Psychiatrie und Psychotherapie leidet.

Wir hoffen, dass wir Sie, Leser der Psychiatrischen Praxis, mit diesem Editorial angeregt haben, über den Stellenwert der Psychotherapie in der Weiterbildung nachzudenken und dafür einzutreten, dass störungsspezifische Psychotherapiemethoden in der Versorgung psychisch kranker Menschen großflächige Anwendung finden.

\section{Literatur}

1 DGPPN, BÄK, KBV, AWMF (Hrsg.). Nationale Versorgungsleitlinie Unipolare Depression. Berlin: Springer; 2011

2 Falkai P, Herpertz S. Entwurf für ein neues Weiterbildungscurriculum für Psychotherapie in der Psychiatrie. Psychiatrie 2012: (im Druck) 